\title{
Hepatitis C virus - from an unknown virus to a curable disease
}

\author{
Raluca Mihaela Dirtu ${ }^{1,2}$, Andreea Ruxandra Cazan ${ }^{1,2}$, George Sebastian Gherlan ${ }^{1,2}$, \\ Simin-Aysel Florescu ${ }^{1,2}$, Emanoil Ceausu ${ }^{1,2}$, Petre lacob Calistru ${ }^{1,2}$ \\ 1"Carol Davila" University of Medicine and Pharmacy, Bucharest, Romania \\ 2"Dr. Victor Babes" Clinical Hospital of Infectious and Tropical Diseases, Bucharest, Romania
}

\begin{abstract}
The infection with hepatitis C virus has drawn attention to researchers from 1970. Nowadays, hepatitis C virus infection is a global public health issue, affecting approximately $3 \%$ of the global population. Experimental models provided vital data on the understanding of viral replication. Chronic infection with hepatitis $\mathrm{C}$ virus is generally a progressive lent disease characterized by the persistence of inflammation in the liver and correlated with the cirrhosis in about $20 \%$ of patients after $20-30$ years of infection. Hepatitis $C$ virus is still a public health threat, but progress in monitoring and treatment of infected patients is truly noticeable and has remarkably improved the prospects for this disease.
\end{abstract}

Keywords: hepatitis C virus, DAA, SVR

\begin{abstract}
Abbreviations
DAA: Direct-acting antivirals

SVR: Sustained virologic response

HCC: Hepatocellular carcinoma

FDA: Food and Drug Administration

WHO: World Health Organization

HALT-C: The Hepatitis C Antiviral Long-term Treatment against Cirrhosis
\end{abstract}

\section{Historical perspective and current concept of infection with hepatitis $C$ virus}

The infection with hepatitis $\mathrm{C}$ virus has drawn attention to researchers from 1970, when Harvey J. Alter (the director of the transfusion department of the USA) noticed that transfused patients acquired a form of hepatitis whose virus is different and unknown and falls within the non-A, non-B hepatitis group $(1,2)$. In 1988, after years of research, Alter confirmed the new virus illustrating his presence in a group of specimens of non-A, non-B hepatitis (2). In April 1989, the first article about hepatitis C virus was published in „Science“ magazine, entitled „Detection of antibody to hepatitis $\mathrm{C}$ virus in pro- spectively followed transfusion recipients with acute and chronic non-A, non-B hepatitis“ (2). Nowadays, hepatitis $C$ virus infection is still a global public health issue, affecting approximately $3 \%$ of the global population $(1,2)$.

Experimental models provided vital data on the understanding of viral replication. Thus, it was found that the virion hacked into cell after interacting with surface receptors such as CD81, LDL receptor, EGF receptor, human scavenger receptor SR-B1, CLDN1, OCLN2 (2). After attachment and penetration, by conformational changes in the endosome membrane, the RNA is released in the cytoplasm (2,3). Direct viral translation occurs in 
combination with rough endoplasmic reticulum (RER) (3). It takes place the synthesis of structural and nonstructural proteins, assembling the replicative and terminal complex of viral RNA, the synthesis of the positive copies of RNA, the assembly of the viral particle in the RER and the Golgi apparatus and release the virus from hepatocytes (3).

\section{The structure of hepatitis $\mathbf{C}$ virus}

Understanding the viral replication mechanisms enabled the development of current interferon-free therapies, based on direct-acting antivirals (DAA) $(2,3)$.

The genome of hepatitis $\mathrm{C}$ virus is extremely unstable, thus resulting in permanent errors, correlated with genetic heterogeneity and the occurrence of the quasispecies in the same infected person $(3,4)$.

The HVR variable region, located at the end of the N-terminal of the E2 protein is responsible for the occurrence of mutations $(3,4)$. Variations in HVR-1 and HVR-2 are generated very quickly and can provide adaptive advantages with regard to viral tropism, virulence or resistance to treatment $(3,4)$. Mutations in non-structural proteins, such as NS5A, provide sensitivity to interferon treatment (IFN) (4). The responsible region is located in the C-terminal portion of the NS5A protein and is called the interferon-sensitive region (ISDR) $(4,5)$.

Chronic infection with hepatitis $\mathrm{C}$ virus is generally a progressive lent disease characterized by the persistence of inflammation in the liver and correlated with the cirrhosis in about $20 \%$ of patients after 20-30 years of infection (progression rate vary between $2 \%$ and $51 \%$ after 22 years of persistent infection) (5).

\section{Natural evolution of virus $\mathrm{C}$ infection}

Once the cirrhosis established, evolution is hard to anticipate and may vary differently to each patient: can remain insolent, perhaps evolving to hepatocellular carcinoma (HCC) may cause decompensated cirrhosis or even death. Thein et al. analyzed 111 reviews, summing 33,000 patients, and highlighted that, once installed cirrhosis, there is an annual risk between 1 and $5 \%$ to develop HCC, an annual risk between 3 and $6 \%$ for decompensated cirrhosis and after decompensation an annual risk between 15 to $20 \%$ of death (5). HCC incidence among chronic infection is $1-3 \%$ to 30 years (5). Once installed cirrhosis, risk is between 1 and $4 \%$ per year, with a higher rate in patients who have acquired the virus following contaminated blood products. Negative prognostic factors are similar to those incriminated in raising the rate of progression of cirrhosis (5). The data obtained from

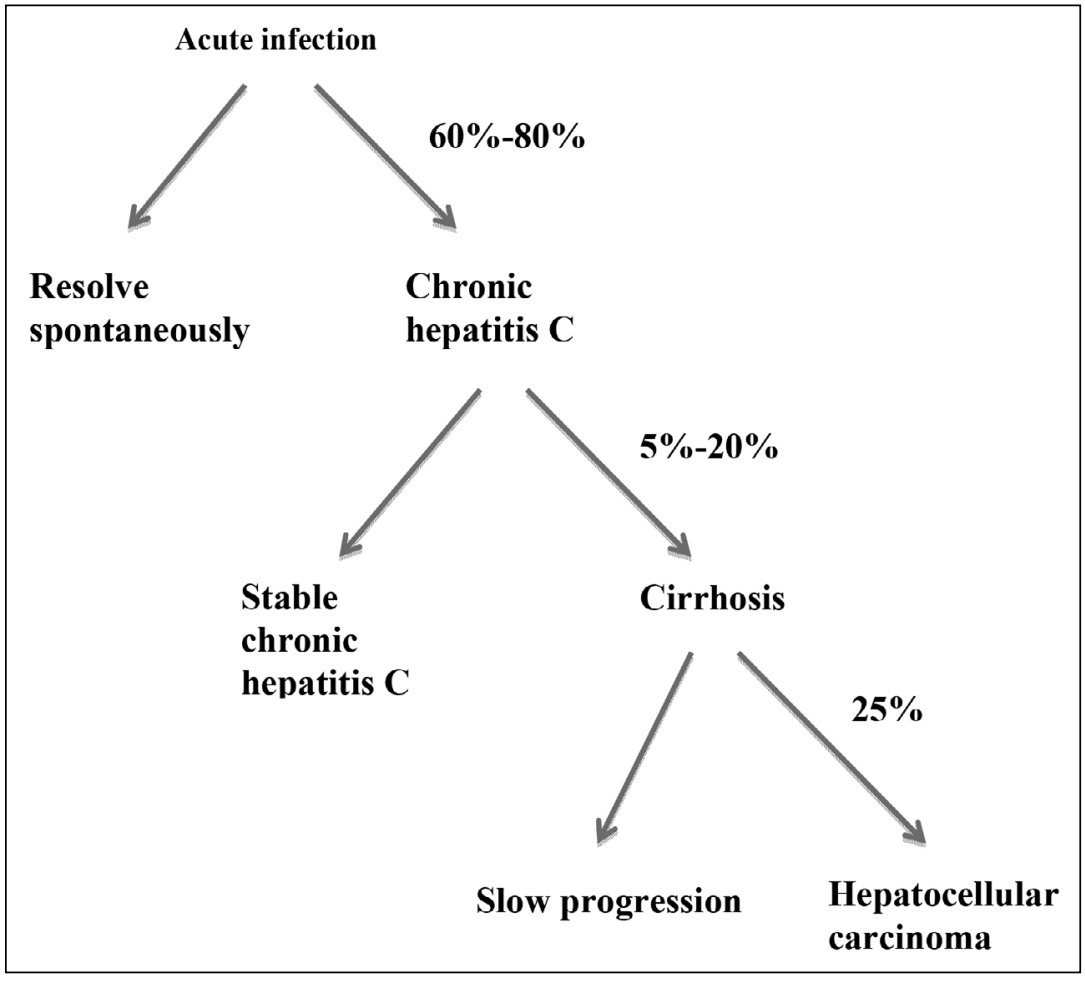

FIGURE 1. Natural evolution of virus $C$ infection (Adapted from "Mandell, Douglas and Bennett's Principles and Practice of Infectious Diseases, $8^{\text {th }}$ Edition", 2015) 
the HALT-C study showed that the HCC development rate in patients with cirrhosis was $7 \%$ after 4.8 years $(5)$.

Numerous studies and meta-analyses illustrated that the elimination of virus $\mathrm{C}$ infection with directacting antivirals therapy reduces the risk of HCC in patients with chronic infection, independent of fibrosis stage. The attainment of the sustained virologic response (SVR) in patients with cirrhosis provides a $20 \%$ reduction in $\mathrm{HCC}$, and in patients with advanced liver disease, SVR has determined the reduction of $\mathrm{HCC}$ development risk from $17.8 \%$ to $4.2 \%$ with a reduction in incidence from $3.3 \%$ per year to $1.05 \%$ per year $(4)$,5 .

The infection with hepatitis $\mathrm{C}$ virus is no longer considered a local disease, but is regarded as a systemic disease, which can also affects many organs and tissues, not just liver $(5,6)$. Around 30-40\% from the patients with persistent infection develop extrahepatic manifestations, such as: lymphoma, cryoglobulinemia, glomerulonephritis, porphyria cutanea tarda, insulin-resistance, diabetes, Sjögren's syndrome, vascular disease (cardiovascular, cerebrovascular) $(5,6)$.

\section{Global distribution of hepatitis $\mathrm{C}$ virus genotypes}

Hepatitis $\mathrm{C}$ virus has spread irregularly around the world, there are low prevalence zones but also „burden“ zones with incidence and prevalence increased (1). The genotypes are distributed differently across the world: in Europe and North America genotype $1(49.1 \%$ of cases) is dominant $(1,7)$. This genotype is divided into two subclasses: 1a and $1 \mathrm{~b}$, the latter being identified both in Europe (more than $68 \%$ of cases) and in Romania (over $99.6 \%$ of cases) $(1,7)$. Genotype 3 is the second most widely spread on the globe with a $17.9 \%$ and $25.5 \%$ percent in Europe (7). Genotype 4 is commonly encountered in North Africa and Middle East, particularly in Egypt (following the injection treatment used for schistosomiasis that infected millions of people with hepatitis $\mathrm{C}$ virus). Genotype 5 is preponderant in South Africa $(1,7)$.

\section{Treatment with DAA - a new therapeutic era}

The therapeutic target of the DAA molecules is the non-structural proteins (NS3/4A, NS5B, NS5A) of the viral genome $(7,8)$. In 2011 , two protease in- hibitors NS3A (boceprevir and telaprevir) were authorized. Thus, the SVR increased from $40 \%$ in patients treated with interferon-only therapy to $75 \%$ at the first generation of DAA $(7,8,9)$. Practically since 2011 , when these molecules were introduced in the treatment of patients with $\mathrm{C}$ virus, the whole world witnessed in real time a medical and therapeutic revolution in the fight against hepatitis $\mathrm{C}$ with virus $(10,11,12)$. In 2013, the second generation of DAA (sofosbuvir) is introduced in combination with ribavirin and peg-interferon in order to improve SVR $(3,13)$. In 2014-2015, the Food and Drug Administration (FDA) approved the combination of ombitasvir/paritaprevir/ritonavir/dasabuvir to treat patients with genotype 1 and the combination of ombitasvir/paritaprevir/ritonavir in a single tablet in combination with ribavirin for the treatment of patients with genotype $4(12,13)$. In 2016, pangenotypic combination sofosbuvir/velpatasvir was approved in combination or not with ribavirin to treat adult patients regardless of genotype (13).

Thus, various studies have drawn conclusions or are still ongoing in order to identify the long-term risks of these treatments $(13,14)$. A study conducted in France (published in „The Lancet" in February 2019) on a cohort of 10,000 patients, in the period 2012-2015, evaluated the incidence of mortality and morbidity, the risk of hepatocellular carcinoma and decompensated cirrhosis in the DAA-treated population compared to the untreated (14). After adjusting all age variables, gender, genotype, fibrosis degree, comorbidities it was concluded that the treatment with DAA decreased the risk of mortality and morbidity by $95 \%$ in those treated compared to untreated (14). In 2018, FDA approved the combination of sofosbuvir/ledipasvir or sofosbuvir plus ribavirin in the population aged 12 to 17 years (14).

\section{Future strategies}

Hepatitis $\mathrm{C}$ virus is still a public health threat, but progress in monitoring and treatment of infected patients is truly noticeable and has remarkably improved the prospects for this disease (15). In 2016, World Health Assembly adopted „The Global Health Sector Strategy on Viral Hepatitis 20162030“, where World Health Organization (WHO) 
members aimed to reach hepatitis elimination by 2030 decreasing the incidence by $90 \%$, reducing

\section{REFERENCES}

1. Mandell, Douglas and Bennett's Principles and Practice of Infectious Diseases, $8^{\text {th }}$ Edition, 2015

2. Alter MJ, Margolis HS, Krawczynski K et al. The natural history of community-acquired hepatitis $C$ in the United States. The Sentinel Counties Chronic non-A, non-B Hepatitis Study Team. N Engl J Med. 1992 Dec 31;327(27):1899-905.

3. Conti F, Buonfiglioli F, Scuteri A et al. Early occurrence and recurrence of hepatocellular carcinoma in HCV-related cirrhosis treated with direct-acting antivirals. J Hepatol. 2016 Oct;65(4):727733.

4. Reig M, Mariño Z, Perelló $C$ et al. Unexpected high rate of early tumor recurrence in patients with $\mathrm{HCV}$-related $\mathrm{HCC}$ undergoing interferon-free therapy. J Hepatol. 2016 Oct;65(4):719-726.

5. Hawkins $C$, Grant J, Ammerman LR et al. High rates of hepatitis $C$ virus (HCV) cure using direct-acting antivirals in HIV/HCV-coinfected patients: a real-world perspective. J Antimicrob Chemother. 2016 Sep;71(9):2642-5.

6. Rachel H. Westbrook, Geoffrey Dusheiko. Natural history of hepatitis C. Journal of Hepatology, 2014.

7. Nicholas J Burstow et al. Hepatitis C treatment: where are we now? Int J Gen Med. 2017 Feb 17;10:39-52.

8. AASLD-IDSA. Recommendations for testing, management, and treating hepatitis $\mathrm{C}$. Monitoring patients who are starting $\mathrm{HCV}$ treatment, are on treatment, or have completed therapy. mortality by $65 \%$ and increasing the diagnostic and treatment rate by over $90 \%$ (15).

Conflict of interest: none declared Financial support: none declared

9. Heimbach JK, Kulik LM, Finn RS, et al. AASLD guidelines for the treatment of hepatocellular carcinoma. Hepatology. 2018;67:358-380.

10. Marcellin F, Roux P, Protopopescu C, Duracinsky M, Spire B, Carrieri MP. Patient-reported outcomes with direct-acting antivirals for the treatment of chronic hepatitis C: current knowledge and outstanding issues. Expert Rev Gastroenterol Hepatol. 2017;11:259-268.

11. Charlton M, Everson GT, Flamm SL, et al. Ledipasvir and sofosbuvir plus ribavirin for treatment of $\mathrm{HCV}$ infection in patients with advanced liver disease. Gastroenterology. 2015;149:649-59.

12. Cacoub $P$, Desbois $A C$, Isnard-Bagnis $C$, Rocatello $D$, Ferri $C$. Hepatitis $C$ virus infection and chronic kidney disease: Time for reappraisal. J Hepatol. 2016;65:S82-S94.

13. Johannes Vermehren et al. Challenges and perspectives of direct antivirals for the treatment of hepatitis $C$ virus infection. $J$ Hepatol. November 2018, volume 69, issue 5, p 1178-1187.

14. Fabrice Carrat et al. Clinical outcomes in patients with chronic hepatitis $\mathrm{C}$ after direct-acting antiviral treatment: a prospective cohort study. The Lancet, February 2018.

15. Guidelines for the care and treatment of the persons diagnosed with chronic hepatitis C virus infection, July 2018. 